\title{
ROLE OF THERMAL FLUCTUATIONS IN THE DAMPING OF THE GIANT DIPOLE RESONANCE OF SPHERICAL AND DEFORMED NUCLEI: ${ }^{90} \mathrm{Zr}$ AND ${ }^{164} \mathrm{Er}^{\text {मे }}$
}

\author{
M. GALLARDO, F.J. LUIS \\ Departamento de Física Atómica y Nuclear, Facultad de Física, Universidad de Sevilla, Apartado 1065, E-41080 Sevilla, Spain
}

and

\author{
R.A. BROGLIA \\ Dipartimento di Fisica, Universita di Milano and INFN Sezione di Milano, Via Celoria 16, I-20133 Milan, Italy \\ and Niels Bohr Institute, University of Copenhagen, Blegdamsvej 17, DK-2100 Copenhagen Ø, Denmark
}

Received 21 January 1987; Revised manuscript received 25 March 1987

\begin{abstract}
We study the damping width of the giant dipole resonance (GDR) built on the ground state and on excited states of ${ }^{90} \mathrm{Zr}$, which is spherical at zero spin and temperature, and ${ }^{164} \mathrm{Er}$. which is deformed. Neither of these nuclei changes appreciably their equilibrium shape when excited. Nonetheless, fluctuations due to finite temperature produce a marked increase in the width of the GDR of ${ }^{90} \mathrm{Zr}$, leaving essentially unchanged that of ${ }^{164} \mathrm{Er}$. This is because in the case of deformed nuclei thermal fluctuations imply the sampling of both larger and smaller deformations around the equilibrium shape, while spherical nuclei can only feel larger distortions. In spite of the stability of the GDR of ${ }^{164} \mathrm{Er}$, the angular distribution of the associated $\gamma$-rays is strongly affected by the temperature.
\end{abstract}

A recent compilation of the properties [1] of the GDR can be found in ref. [2]. Fig. 17 of this reference displays the observed width (FWHM) of the associated strength functions based on the ground state and on excited states for a number of nuclei, as a function of the mass number in the range $40 \lesssim A \lesssim 160$. At $E^{*}=0$ the data displays a marked structure. The minima $(\Gamma \simeq 4 \mathrm{MeV})$ correspond to closed shell nuclei while the maxima $(\Gamma \lesssim 8 \mathrm{MeV})$ are associated with deformed nuclei. This structure is washed out in the case of giant resonances built on excited states. In fact, the data shows a uniform value around $\Gamma \simeq 8 \mathrm{MeV}$, implying that the GDR of nuclei that are spherical in the ground state have become considerably broader, while those corresponding to well-deformed nuclei remain approximately unchanged.

\footnotetext{
4his work is partially supported by the CAICYT (Spain) under contract number $2868 / 83$ and by a grant of the Commemortive Association for the Japan World Exposition.
}

The main mechanism responsible for the damping of giant resonances in medium and heavy nuclei arises from the coupling to low-lying surface oscillations [3]. A static quadrupole deformation as one limit of this mechanism (adiabatic limit), produces a breaking of the giant dipole resonance and thus a broadening of the associated distribution [4].

Angular momentum ( $I \lesssim 50 \hbar$ ) leads, as a rule, to the deformation of spherical nuclei, without essentially changing the shape of well-deformed nuclei [5-7]. It is thus tempting to conclude that this is the reason why the strength function of GDR observed in the $\gamma$-decay of compound nuclei have a constant width as a function of mass number.

In the present paper we check this conjecture by studying the photoabsorption cross section for two extreme cases in the compilation of ref. [2]: ${ }^{90} \mathrm{Zr}$, spherical in the ground state and ${ }^{164} \mathrm{Er}$, which is well deformed. We compare the distribution of the dipole strength based on the ground state and on the com- 
pound nucleus state, defined by the experimental conditions

$$
\begin{aligned}
& { }^{90} \mathrm{Zr}: \quad T=0, I=0 \text { and } T=1.5 \mathrm{MeV}, I=7.5 \hbar, \\
& { }^{164} \mathrm{Er}: \quad T=0, I=0 \text { and } T=1.2 \mathrm{MeV}, I=21 \hbar .
\end{aligned}
$$

The calculations were carried out as in ref. [8]. A cranked Nilsson potential, with the parameters taken from ref. [6] for ${ }^{90} \mathrm{Zr}$ and from ref. [9] for ${ }^{164} \mathrm{Er}$, is used as the single-particle field. For ${ }^{90} \mathrm{Zr}$ these parameters give a fair dipole response at $E^{*}=0$ where most of the strength is concentrated in one peak as experimentally observed. On the other hand, all the sets of parameters we used for ${ }^{164} \mathrm{Er}$ yield an anomalous dipole strength splitting at low energies ${ }^{\ddagger 1}$. The set given in ref. [9] is in any case the best one available at present for this mass region.

Pairing is not included in the calculations. At the temperatures of interest its effect is expected to be small [10]. For non-zero temperatures, the occupation parameters of the single-particle levels are given by the Fermi distribution [11]. The residual interaction is approximated by a separable dipole-dipole force. The coupling strengths are chosen to fit the experimental energy centroids of the GDR at $T=0, I=0\left(\kappa / \kappa_{\mathrm{sc}}=0.765,0.697\right.$ for ${ }^{164} \mathrm{Er}$ and ${ }^{90} \mathrm{Zr}$, where the self-consistent values [4] are $\kappa_{\text {sc }}=0.102,0.274 \mathrm{MeV} / \mathrm{fm}^{2}$, respectively).

The dipole cross section in the laboratory frame $\sigma$ is calculated in the linear response theory $[12,13]$ (cf. also ref. [8] and note an error of a missing factor $1 / 3$ in eqs. (12) and (15)). Thermal fluctuations of the nuclear shape are included in the calculations. Thus the total cross section is written as [8]

$$
\begin{aligned}
& \langle\sigma(I, T)\rangle ; \\
& \frac{\int \epsilon \mathrm{d} \epsilon \mathrm{d} \gamma P(\epsilon, \gamma ; I, T) \sigma(\epsilon, \gamma ; I, T)}{\int \epsilon \mathrm{d} \epsilon \mathrm{d} \gamma P(\epsilon, \gamma ; I, T)},
\end{aligned}
$$

where

$$
P(\epsilon, \gamma ; I, T) \sim \exp [-F(\epsilon, \gamma ; I, T) / T] .
$$

\footnotetext{
F1 We note that the unperturbed dipole response in the ground state needs to be studied more carefully. All the realistic singleparticle potentials give, in general, a bad description of it.
}

The quantity $F(\epsilon, \gamma ; I, T)$ is the free energy for a given temperature $T$, spin $I$ and deformation parameters $(\epsilon, \gamma)$. No hexadecapole deformations are considered. The free energy surfaces $F$ are calculated with the Strutinsky method [7]. Fig. 1 shows these surfaces for the two nuclei under study and for the values of $T$ and $I$ displayed in (1). The equilibrium deformations remain in both cases essentially unchanged. Consequently, the observed broadening of the GDR in ${ }^{90} \mathrm{Zr}$ is not due to a change in shape, as was the case in ${ }^{108} \mathrm{Sn}$ [8], but must arise from the sampling of finite distortions under the influence of thermal fluctuations.

For systems which display non-spherical equilibrium shapes at $T, I \neq 0$ like ${ }^{164} \mathrm{Er}$, the role of the shape fluctuations is not so important. The thermal ensemble includes, with similar weights, both smaller and larger deformations than the one associated with equilibrium, the summed effect being small.

This picture is borne out by the results of the calculations displayed in fig. 2 . There is a significant increase in the width of the GDR of ${ }^{90} \mathrm{Zr}$ with excitation energy. On the other hand, the dipole strength of ${ }^{164} \mathrm{Er}$ displays two peaks and thus a sizeable width at $T=I=0$. This is because ${ }^{164} \mathrm{Er}$ has a stable deformation $\left(\epsilon=0.26, \gamma=0^{\circ}\right)$ in the ground state. At $T=1.2 \mathrm{MeV}$ and $I=21 \hbar$, both peaks become somewhat broader due to the averaging effect of thermal fluctuations. The total width is, however, little affected being still controlled by the separation between the peaks and reflecting the most probable static deformation $\left(\epsilon=0.19, \gamma=0^{\circ}\right)$.

Calculations of the GDR using a pure harmonic oscillator potential as the single-particle hamiltonian [14] are shown in fig. 3. The associated results do not display Landau damping. Consequently, the effects of rotation and temperature appear clearer in this simpler model.

To obtain a fair description of the GDR in the ground state, the results of the calculations were folded by a lorentzian function with an empirical energy dependent width ${ }^{\ddagger 2}$ [15]

\$2 Because of the strong Landau damping present in the calculations with the Nilsson potential, the folding procedure will not improve the results. Therefore we have not performed it in that case. 

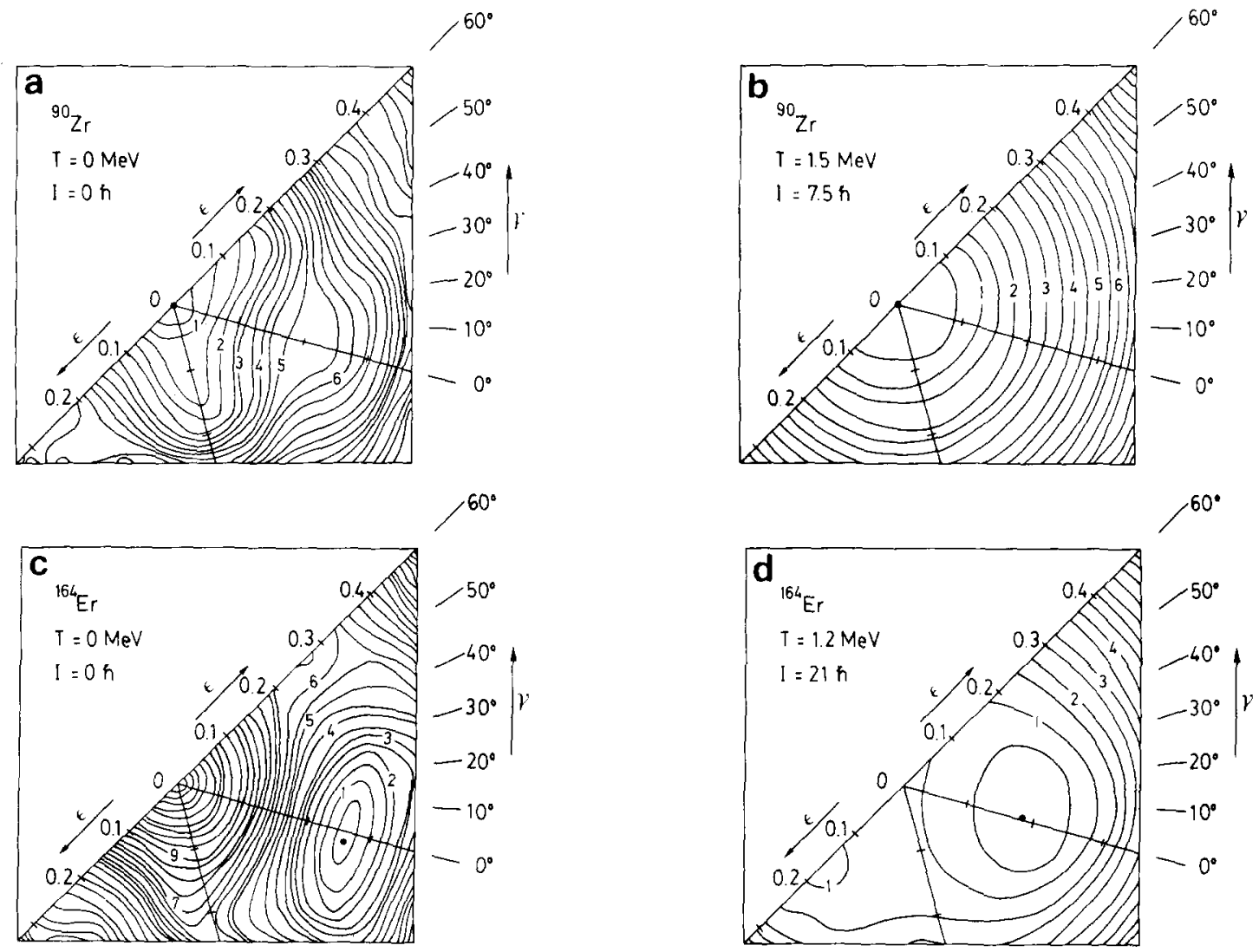

Fig. 1. Strutinsky free energy surfaces as function of the deformation parameters for ${ }^{90} \mathrm{Zr}$ at $T=I=0$ (a) and at $T=1.5 \mathrm{MeV}, I=7.5 \hbar$ (b) and ${ }^{164} \mathrm{Er}$ at $T=I=0$ (c) and $T=1.2 \mathrm{MeV}, I=21 \hbar$ (d). The equipotential lines are represented in steps of $0.5 \mathrm{MeV}$ away from the minimum which is denoted by a full dot.

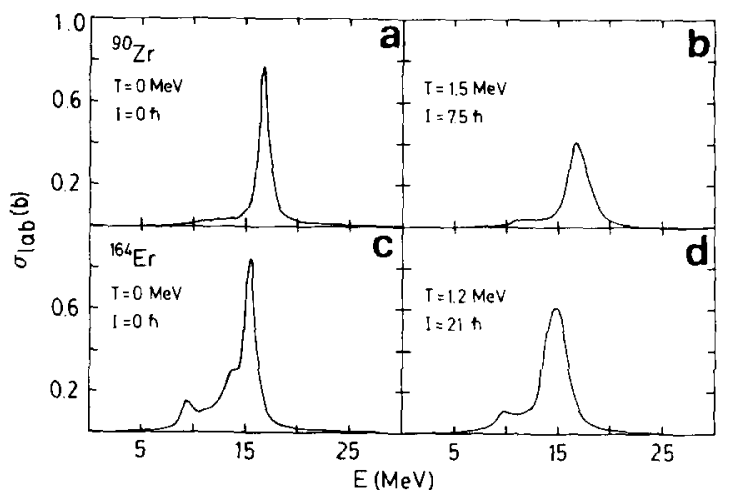

Fig. 2. Photoabsorption cross section in the laboratory system for the GDR of ${ }^{90} \mathrm{Zr}$ and ${ }^{164} \mathrm{Er}$ at the conditions given in (1). A cranked Nilsson potential is used as the single-particle hamiltonian. for $T \neq 0$ the total cross section is calculated according to eq. (2).
$\Gamma=\Gamma_{0}\left(E / E_{\mathrm{c}}\right)^{1.9}$

The quantity $\Gamma_{0}$ is chosen to fit the experimental data at $T=I=0$, while $E_{\mathrm{c}}$ is the energy centroid of the dipole strength distribution. These parameters are kept fixed throughout the calculations. This is somewhat equivalent to assuming that the effect of the coupling of $2 \mathrm{p}-2 \mathrm{~h}$ states to the giant vibration does not change neither with temperature [16] nor with spin.

In figs. $3 a, 3 b$ and $3 d, 3 e$ we compare the GDR built on the equilibrium deformation at zero and nonzero excitation energies. The thermal shape fluctuations are included in the results reported in figs. 3c and $3 \mathrm{f}$ for ${ }^{90} \mathrm{Zr}$ and ${ }^{164} \mathrm{Er}$, respectively. A resume of the different widths is collected in table 1 .

Similar comments to those made in connection 

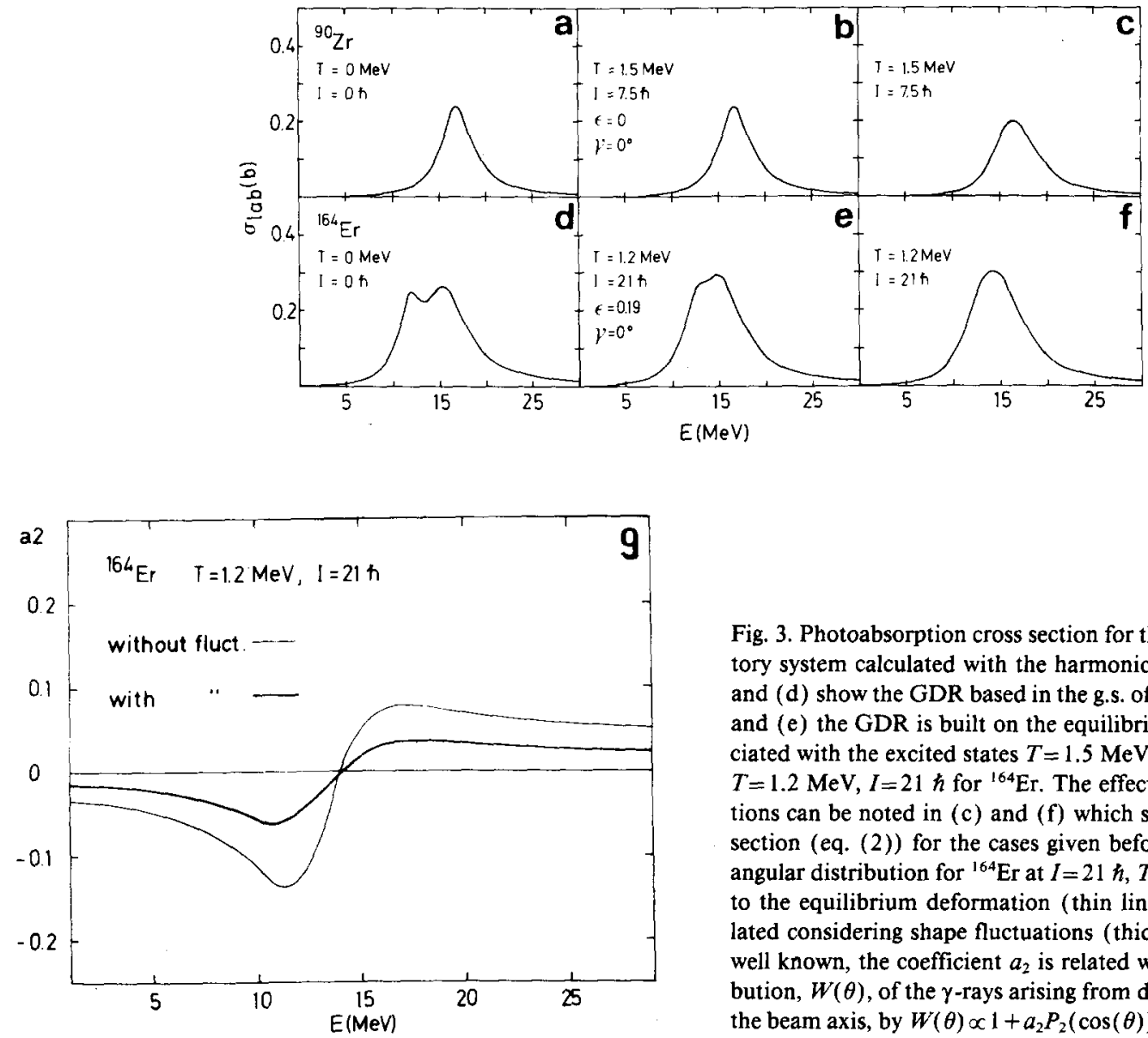

Fig. 3. Photoabsorption cross section for the GDR in the laboratory system calculated with the harmonic oscillator model. (a) and (d) show the GDR based in the g.s. of ${ }^{90} \mathrm{Zr}$ and ${ }^{164} \mathrm{Er}$. In (b) and (e) the GDR is built on the equilibrium deformation associated with the excited states $T=1.5 \mathrm{MeV}, I=7.5 \hbar$ for ${ }^{90} \mathrm{Zr}$ and $T=1.2 \mathrm{MeV}, I=21 \hbar$ for ${ }^{164} \mathrm{Er}$. The effect of the shape fluctuations can be noted in (c) and (f) which show the average cross section (eq. (2)) for the cases given before. (g) represents the angular distribution for ${ }^{164} \mathrm{Er}$ at $I=21 h, T=1.2 \mathrm{MeV}$ associated to the equilibrium deformation (thin line) and the one calculated considering shape fluctuations (thick line). (As it is very well known, the coefficient $a_{2}$ is related with the angular distribution, $W(\theta)$, of the $\gamma$-rays arising from dipole decay relative to the beam axis, by $W(\theta) \propto 1+a_{2} P_{2}(\cos (\theta))$.)

Table 1

Damping width (FWHM) of a variety of giant dipole resonances. In columns 4 and 5 the ratios between the width $\Gamma$ of the GDR response function based on a state with temperature $T$ and angular momentum $I$ and on the ground state are reported (cf. fig. 3 ). The calculations for the case of ${ }^{108} \mathrm{Sn}$ were carried out folding the associate dipole response function taken from ref. [8] with a lorentzian function of width $\Gamma=5 \mathrm{MeV}$ which is assumed to simulate the coupling to $2 \mathrm{p}-2 \mathrm{~h}$ states. In the last two columns the width $\Gamma(0)$ of the GDR response function based on the ground state is shown. The calculations for ${ }^{90} \mathrm{Zr}$ and ${ }^{108} \mathrm{Sn}$ are obtained eliminating the effect due to Landau damping of the result of ref. [3]. where the coupling of the giant vibration to $2 \mathrm{p}-2 \mathrm{~h}$ states containing a surface vibration is considered. The width of the GDR of ${ }^{164} \mathrm{Er}$ results from the convolution of the strength function resulting from the calculation of the vibration in the deformed potential $(\epsilon=0.26)$ and a lorentzian width $\Gamma=\Gamma_{0}\left(E / E_{\mathrm{c}}\right)$ (cf. the text for more details).

\begin{tabular}{|c|c|c|c|c|c|c|}
\hline \multirow[t]{2}{*}{ Nucleus } & \multirow[t]{2}{*}{$T(\mathrm{MeV})$} & \multirow[t]{2}{*}{$I(h)$} & \multicolumn{2}{|l|}{$\Gamma\left(\mathrm{E}^{*}\right) / \Gamma(0)$} & \multicolumn{2}{|l|}{$\Gamma(0)(\mathrm{MeV})$} \\
\hline & & & experiment & theory & experiment & theory \\
\hline${ }^{90} \mathrm{Zr}$ & 1.5 & 7.5 & 2 & 1.4 & 4 & 4.6 \\
\hline${ }^{108} \mathrm{Sn}$ & 2 & 60 & 2.1 & 1.9 & 5 & 5 \\
\hline${ }^{164} \mathrm{Er}$ & 1.2 & 21 & 1 & 0.9 & 8.2 & 7 \\
\hline
\end{tabular}


with fig. 2 also apply here. To be added is that even if the absorption cross sections for ${ }^{164} \mathrm{Er}$ when thermal fluctuations are not included (fig. 3e) and when they are included (fig. 3f) are very similar, the $\gamma$ decay angular distribution shows a profound change in the last case. This is apparent in fig. $3 \mathrm{~g}$ where the coefficient $a_{2}$ is plotted. It is calculated from the expression

$a_{2}=\frac{1}{4}\left(3 \sigma_{10} / \sigma-1\right)$

where $\sigma$ is the total dipole absorption cross section and $\sigma_{10}$ the one associated with the dipole transitions carrying no angular momentum with respect to the axis of rotation (assuming total spin $I \gg 1 \hbar$ ).

The thermal averaged result (thick line of fig. $3 \mathrm{~g}$ ), calculated from the corresponding dipole cross section, displays overall agreement with the experimental data [17]. It implies a diminution of the anisotropy with respect to the case with no fluctuations (thin line), although a prolate shape of the nucleus can still be suggested. It is likely that the study of the $\gamma$-ray angular distributions is the specific tool to learn about the surface thermal fluctuations of the compound nucleus [18].

We conclude that the marked variations of the width of GDR recorded as a function of temperature and angular momentum are due to changes in deformation and/or to thermal shape fluctuations. The effect of the Coriolis force is not found to be important. From the knowledge of the free energy surfaces as a function of $I$ and $T$ it seems thus possible to infer the associated evolution of the photoabsorption cross section.

\section{References}

[1] J.E.Draper et al., Phys. Rev. Lett. 49 (1982) 434; J. Barrete and A.M. Sandorfi, Comm. Nucl. Part. Phys. 12 (1983) 57

B. Haas et al., Phys. Lett. B 120 (1983) 79; W. Hennerici et al., Nucl. Phys. A 396 (1983) 329; A.M. Sandorfi et al., Phys. Lett. B 130 (1983) 19; L. Lazzarini et al., Phys. Rev. Lett. 53 (1984) 1045; J.J. Gaardhøje et al., Phys. Lett. B 139 (1984) 273; J.J. Gaardhøje et al., Phys. Rev. Lett. 53 (1984) 148;
D. Schwalm et al., Frontiers in nuclear dynamics, eds. R.A Broglia and C.H. Dasso (Plenum, New York, 1985) p. 33. C.A. Gosset et al., Phys. Rev. Lett. 54 (1985) 1486; Th. Arctaedius et al., Phys. Lett. B 158 (1985) 205; J.J. Gaardhøje et al., Nuclear structure 1985, eds. R.A. Broglia, G. Hagemann and B. Herskind (North-Holland, Amsterdam, 1985) p. 519.

[2] K.A. Snover, Annu. Rev. Nucl. Part. Sci. 36 (1986) 1.

[3] P.F. Bortignon and R.A. Broglia, Nucl. Phys. A 371 (1981) 405 ;

G.F. Bertsch, P.F. Bortignon and R.A. Broglia, Rev. Mod. Phys. 55 (1983) 287

[4] A. Bohr and B. Mottelson, Nuclear structure, Vol II (Benjamin, New York, 1985).

[5] G. Andersson, S.E. Larsson, G. Leander, P. Moller, S.G. Nilsson, I. Ragnarsson, S. Aberg, R. Bengtsson, J. Dudek, B. Nerlo-Pomorska, K. Pomorsky and Z. Szymanski, Nucl. Phys. A 268 (1976) 205

[6] S. Aberg, Phys. Scr. 25 (1982) 113.

[7] M. Diebel, K. Albrecht and R.W. Hasse, Nucl. Phys. A 355 (1981) 165

[8] M. Gallardo, M. Diebel, T. Døssing and R.A. Broglia, Nucl. Phys. A 443 (1985) 415.

[9] R. Bengtsson and I. Ragnarsson, Nucl. Phys. A 436 (1985) 14.

[10] O. Civitarese, G.G. Dussel and R.P.J. Perazzo, Nucl. Phys. A 404 (1983) 15.

P. Ring, J. Phys. (Paris) C6, Suppi. 6, t. 45 (1984) 247.

[11] A. Bohr and B. Mottelson, Nuclear structure, Vol. I (Benjamin, New York, 1968);

C. Fiolhais and J. da Providencia, Nucl. Phys. A 435 (1985) 190.

[ 12 ] J.L. Egido and P. Ring, Phys. Rev. C 25 (1982) 3239; Nucl. Phys. A 388 (1982) 191;

J.L. Egido, H.J. Mang and P. Ring, Nucl. Phys. A 339 (1980) 390

P. Ring, L.M. Robledo, J.L. Egido and M. Faber, Nucl. Phys. A 419 (1984) 261;

P. Ring, Nucl. Phys. A 421 (1984) 205.

[13] M. Faber, J.L. Egido and P. Ring, Phys. Lett B 127 (1983) 5 , and references therein.

[14] K. Neergard, Phys. Lett. B 110 (1982) 7.

[15] P. Carlos, R. Bergere, A. Beil, A. Lapetre and A. Veyssiere, Nucl. Phys. A 219 (1974) 61;

K.A. Snover, private communication.

[16] P.F. Bortignon, R.A. Broglia, G.F. Bertsch and J. Pacheco, Nucl. Phys. A 460 (1986) 149.

[17] J.A. Behr, G. Feldman, C.A. Gosset, J.H. Gundlach and K.A. Snover, Annual report, University of Washington (1985).

[18] A. Bracco, M. Gallardo, J.J. Gaardhøje and R.A. Broglia, to be published:

M. Gallardo and R.A. Broglia, to be published. 